\title{
CMOS Temperature Sensor with Programmable Temperature Range for Biomedical Applications
}

\author{
Agung Setiabudi ${ }^{1}$, Hiroki Tamura ${ }^{2}$, and Koichi Tanno ${ }^{3}$ \\ ${ }^{1}$ Department of Materials and Informatics, University of Miyazaki, Japan \\ ${ }^{2}$ Department of Environmental Robotics, University of Miyazaki, Japan \\ ${ }^{3}$ Department of Electrical and System Engineering, University of Miyazaki, Japan
}

\begin{abstract}
Article Info
Article history:

Received Dec 11, 2017

Revised Jan 20, 2018

Accepted: Feb 19, 2018

\section{Keyword:}

Biomedical Application

Programmable

Temperature Sensor

Digital

CMOS

Abstract

A CMOS temperature sensor circuit with programmable temperature range is proposed for biomedical applications. The proposed circuit consists of temperature sensor core circuit and programmable temperature range digital interface circuit. Both circuits are able to be operated at $1.0 \mathrm{~V}$. The proposed temperature sensor circuit is operated in weak inversion region of MOSFETs. The proposed digital interface circuit converts current into time using Current-to-Time Converter (ITC) and converts time to digital data using counter. Temperature range can be programmed by adjusting pulse width of the trigger and clock frequency of counter. The proposed circuit was simulated using HSPICE with 1P, 5M, 3-wells, 0.18$\mu \mathrm{m}$ CMOS process (BSIM3v3.2, LEVEL53). From the simulation of proposed circuit, temperature range is programmed to be $0{ }^{\circ} \mathrm{C}$ to $100{ }^{\circ} \mathrm{C}$, it is obtained that resolution of the proposed circuit is $0.392{ }^{\circ} \mathrm{C}$ with $-0.89 /+0.29{ }^{\circ} \mathrm{C}$ inaccuracy and the total power consumption is $22.3 \mu \mathrm{W}$ in $25^{\circ} \mathrm{C}$.
\end{abstract}

Low-power

Low-voltage.
Copyright $(2018$ Institute of Advanced Engineering and Science. All rights reserved.

\section{Corresponding Author:}

Koichi Tanno

Department of Electrical and System Engineering, University of Miyazaki

1-1 Gakuenkibanadai-nishi, Miyazaki, 889-2192, Japan

tanno@cc.miyazaki-u.ac.jp

\section{INTRODUCTION}

Engineering is an innovative field that its origin ideas leading to everything, including biology and medical area. Application of engineering in biology and medical area is then called biomedical engineering. The purpose of this field is combining the design and problem solving skills of engineering with medical and biology sciences to advance health care treatment, including diagnosis, monitoring, and therapy [1]. In the recent years, there is a lot of research focus on biomedical engineering. This topic is becoming interesting and challenging due to the increase of system complexity, human population and its distribution.

One of the most important components in biomedical engineering is sensor. This component is very important because it is directly connected to physical phenomenon [2,3]. In many biomedical applications, sensor plays a crucial role to collect data from many objects such as human body temperature, environment humidity and oxygen levels in the air. The often utilized sensor in biomedical applications is temperature sensor, because temperature is a highly important parameter to monitor, identify, or control many conditions in biomedical field, such as diagnosing human disease, temperature monitoring in operating rooms and preventing bacteria growth in some places. By knowing the useful of temperature sensor in many biomedical applications, the availability of a temperature sensor which can be used in various biomedical applications is highly recommended. The problem is different biomedical application has different temperature range to be measured. It means that the sensor must have wide temperature range. However, wide temperature range causes the resolution of its digital data decreases. To keep the resolution high, high-bit analog to digital (ADC) must be applied. Nevertheless, it will decrease the speed and increase the power consumption. Furthermore, in biomedical applications, not only high sensitivity temperature sensor is needed but also low voltage and low power temperature sensor is strongly required [4], [5], [6].

A low power and low voltage temperature sensor has been proposed and reported [7], [8]. This sensor in 
general achieves low power consumption, but it has small sensitivity. The other high sensitivity temperature sensors are also proposed [9], [10]. However, these sensors are not low voltage and it consumes high power. The other problem is that many conventional on-chip temperature sensor circuits use BJT $\left(V_{b e}\right)$ to sense temperature [4], [9], [11], [12]. The problem of BJT is not able to be implemented in the same chip in many standard CMOS processes. This means that cost of fabrication will increase. The last problem is that many cores of temperature sensor circuits use external bias circuits or high value resistor [8], [9], [13]. These require large chip areas. A low voltage and low power temperature sensor circuit with digital output for health care monitoring system has been proposed in [14]. This sensor can achieve low voltage, low power, high sensitivity and high resolution. However, this sensor is special for health care monitoring system whose temperature range only $33{ }^{\circ} \mathrm{C}$ to $45^{\circ} \mathrm{C}$, and it can not to be used for other biomedical applications with different temperature range.

This paper proposes a new temperature sensor circuits based on previous work [14] with addition of programmable temperature range digitalization. In this paper, the analysis of the circuits, simulation results and the measurement of fabricated temperature sensor core are reported in detail. This paper is organized as follows. The previous research and improvement are presented in Sect. 2. In Sect. 3, the simulation results of the designed circuit and measurement of fabrication temperature sensor core are presented. Finally, Sect. 4 concludes this paper.

\section{PREVIOUS RESEARCH AND IMPROVEMENT}

In this section, the previous research [14] and its improvement are presented in detail. Fig. 1 shows the block diagram of the proposed temperature sensor in previous research. This proposed temperature sensor consists of some sub circuits: sensor core circuit [3], voltage to current converter (VIC) circuit, 1/x circuit, current to time converter (ITC) circuit [15], and counter.

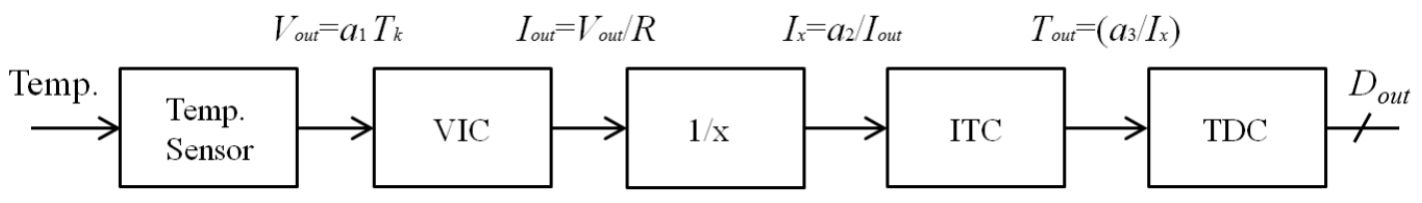

Figure 1. Block diagram of the proposed integrated temperature sensor with digital output

\subsection{Temperature Sensor Core}

Figure 2 shows the temperature sensor core circuit. The temperature sensor core implementation consists of sensor block and start-up block. The start-up block $\left(M_{s 1}, M_{s 2}\right.$ and $\left.M_{s 3}\right)$ is circuit to force turn on the sensor block, and this block does not affect the output voltage. The sensor block is constructed using $M_{1}-M_{5}$. Where, $M_{1}, M_{2}$ and $M_{3}$ are operated in weak inversion region, whereas $M_{4}$ and $M_{5}$ can be operated in both weak and strong inversion region. $I_{d s}$ of the MOSFET operate in the weak inversion region is represented by the following equation.

$$
\begin{gathered}
I_{d s}=I_{0} \frac{W}{L} \exp \left(\frac{V_{g s}-V_{t h}+\eta V_{d s}}{n V_{\theta}}\right)\left(1-\exp \frac{-V_{d s}}{V_{\theta}}\right) \\
I_{0}=2 n \mu C_{o x} V_{\theta}^{2} \\
V_{\theta}=\frac{k}{q} T_{k} \\
n=1+\frac{C_{d}}{C_{o x}}
\end{gathered}
$$

Where $V_{\theta}$ is the thermal voltage, $k\left(=1.38 \times 10^{23} \mathrm{~J} / \mathrm{K}\right)$ is the Boltzmann's constant, $q\left(=1.60 \times 10^{19} \mathrm{C}\right)$ is the electron charge, $T_{K}$ is the absolute temperature, $n$ is slope factor, $\eta$ is DIBL (Drain Induced Barrier Lowering) coefficient, $\mu$ is carrier mobility, $C_{d}$ is capacitance of the depletion layer, $C_{o x}$ is capacitance of the oxide layer.

In (1), if $V_{d s} \geq 4 V_{\theta}, 1 \gg \exp \left(V_{d s} / \mathrm{V}_{\theta}\right)$ is satisfied, as the results $\exp \left(V_{d s} / \mathrm{V}_{\theta}\right)$ can be ignored. Furthermore, because it is well-known that $\eta V_{d s}$ is small value, $\left(V_{g s} V_{t h}\right) \gg \eta V_{d s}$ can be satisfied. From these conditions, (1) can be rewritten to (5).

$$
I_{d s}=I_{0} \frac{W}{L} \exp \left(\frac{V_{g s}-V_{t h}}{n V_{\theta}}\right)
$$




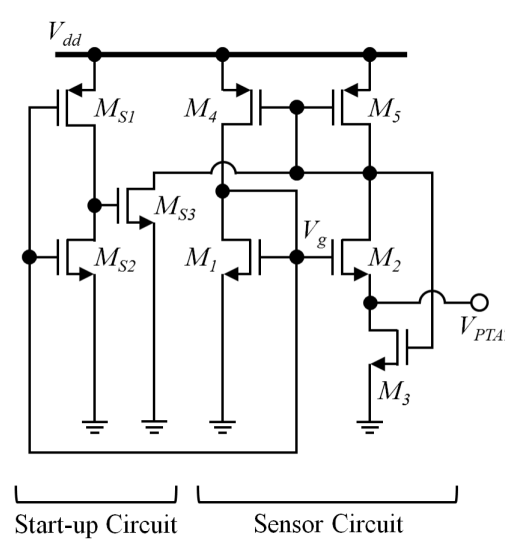

(a)

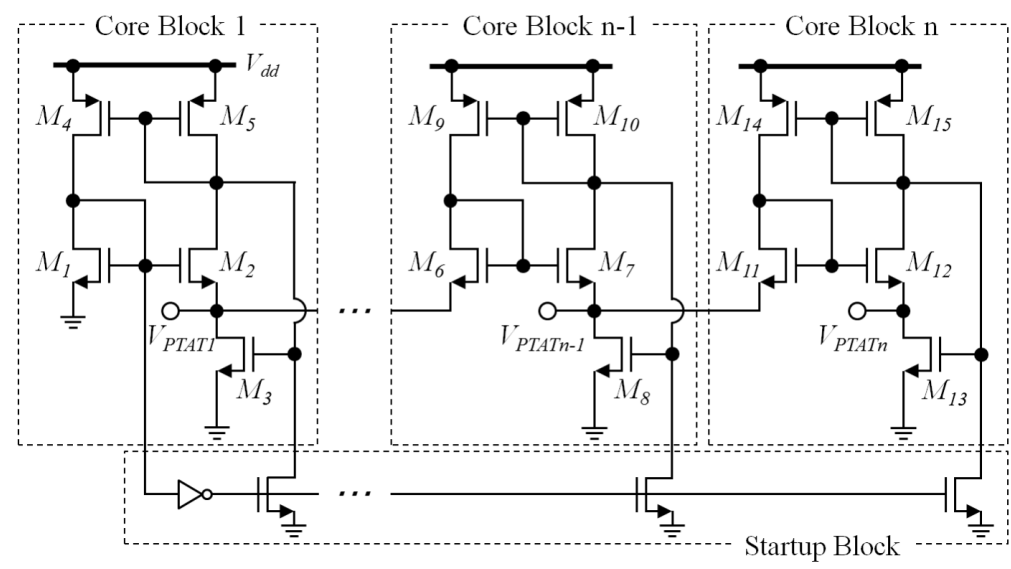

(b)

Figure 2. Temperature Sensor: (a) Core circuit, (b) Cascade connection of temperature sensor core

$$
V_{P T A T 1}=n V_{\theta} \ln \left(\frac{W_{2} / L_{2}}{W_{1} / L_{1}} \cdot \frac{I_{1}}{I_{2}}\right)+V_{t h 1}-V_{t h 2}
$$

And the threshold voltage with the body effect of MOSFET is generally given by (7).

$$
V_{t h}=V_{t h 0}+\gamma\left(\sqrt{\left|2 \Phi_{F}+V_{s b}\right|}-\sqrt{\left|2 \Phi_{F}\right|}\right)
$$

Where $V_{t h 0}$ is the zero-bias threshold voltage, $\gamma$ is the body effect coefficient, $2 \Phi_{F}$ is the surface potential parameter. If $V_{s b}$ is small enough, (7) can be approximated as follows.

$$
V_{t h} \approx V_{t h 0}+\gamma\left(\frac{V_{s b}}{2 \sqrt{2 \Phi_{F}}}\right)
$$

From Fig. 2 (a) it can be known that $V_{s b 1}=0$ and $V_{s b 2}=V_{P T A T 1}$, then $V_{P T A T 1}$ can be expressed as by (9)

$$
V_{P T A T 1}=\frac{n V_{\theta} \ln \left(\frac{W_{2} / L_{2}}{W_{1} / L_{1}} \cdot \frac{I_{1}}{I_{2}}\right)}{1+\gamma\left(\frac{1}{2 \sqrt{2 \Phi_{F}}}\right)}
$$

It is assumed that $\gamma=0.61$ and $2 \Phi_{F}=0.7$, the denominator of (9) is calculated as follows

$$
1+\gamma\left(\frac{1}{2 \sqrt{2 \Phi_{F}}}\right)=1.365 \equiv n^{\prime}
$$

Slope factor $n$ is known to be a value of approximately 1.5, therefore, it can be considered that $n$ ' is relatively close to the value of $n$. As a result, $V_{P T A T 1}$ can be expressed by equation (11).

$$
V_{P T A T 1}=\frac{n}{n^{\prime}} V_{\theta} \ln \left(\frac{W_{2} / L_{2}}{W_{1} / L_{1}} \cdot \frac{I_{1}}{I_{2}}\right) \approx V_{\theta} \ln \left(\frac{W_{2} / L_{2}}{W_{1} / L_{1}} \cdot \frac{I_{1}}{I_{2}}\right) \approx \frac{k}{q} \ln \left(\frac{W_{2} / L_{2}}{W_{1} / L_{1}} \cdot \frac{I_{1}}{I_{2}}\right) T_{K}
$$

From (11), it can be found that $V_{P T A T 1}$ of the proposed circuit is directly proportional to $T_{K}$. Moreover, it could be understood that the output voltage of Fig. 2 is proportional to absolute temperature.

The sensitivity of the sensor can then be increased using cascade connection of the circuit. Fig. 2 (b) shows the cascade connection of circuit Fig. 2 (a). Using (11), $V_{P T A T n}$ of Fig. 2 (b) is as follows.

$$
V_{P T A T n}=\frac{k}{q} \ln \left(\frac{W_{12} / L_{12}}{W_{11} / L_{11}} \cdot \frac{I_{11}}{I_{12}}\right) T_{K}+V_{P T A T n-1}
$$

Using (11) and (12), VPTAT2 is then given by

$$
V_{P T A T n}=\frac{k}{q} \ln \left\{\left(\frac{W_{2} / L_{2}}{W_{1} / L_{1}} \cdot \frac{I_{1}}{I_{2}}\right)+\ldots+\left(\frac{W_{12} / L_{12}}{W_{11} / L_{11}} \cdot \frac{I_{11}}{I_{12}}\right)\right\} T_{K}
$$

From (13), since the output voltage can be expressed by summation of the logarithmic term, this method can be used to increase sensitivity effectively. 


\subsection{Voltage to Current Converter (VIC)}

Figure 3 shows Voltage to Current Converter (VIC). From the figure it can be inferred that $V_{m}=V_{i n}$, and hence the following equation can be obtained.

$$
I_{R}=\frac{V_{i n}}{R}
$$

$I_{R}$ is then copied by two current mirrors $\left(M_{v i 1}, M_{v i 3}, M_{v i 4}\right.$, and $\left.M_{v i 5}\right)$ to $I_{P T A T}$. If the ratio of $W / L$ between $M_{v i 1}$

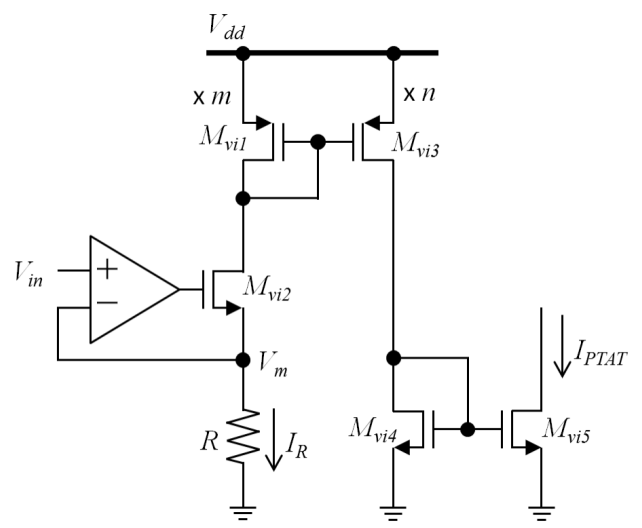

Figure 3. Voltage to Current Converter (VIC)

and $M_{v i 3}$ are $m$ and $n$, and $M_{v i 4}$ and $M_{v i 5}$ are identical, then the following equation can be obtained.

$$
I_{P T A T}=\frac{n}{m} \frac{V_{i n}}{R}
$$

\subsection{1/x Circuit and Current to Time Converter (ITC)}

Figure 4 shows the $1 / x$ circuit and ITC. The $1 / x$ circuit is formed using $M_{t 1}, M_{t 2}, M_{t 3}$, and $M_{t 4}$ which are operated in weak inversion region. Based on translinear principle in weak inversion region $I_{a}$ can be given by the following equation.

$$
I_{a}=\frac{I_{r e f}^{2}}{I_{P T A T}}
$$

Where $I_{r e f}$ is current source which have no temperature dependency, $V_{b}$ is supplied by reference voltage circuit, and $I_{P T A T}$ is the output current of voltage to current converter. The temperature dependence of $I_{a}$ is very small because it utilizes translinear principle.

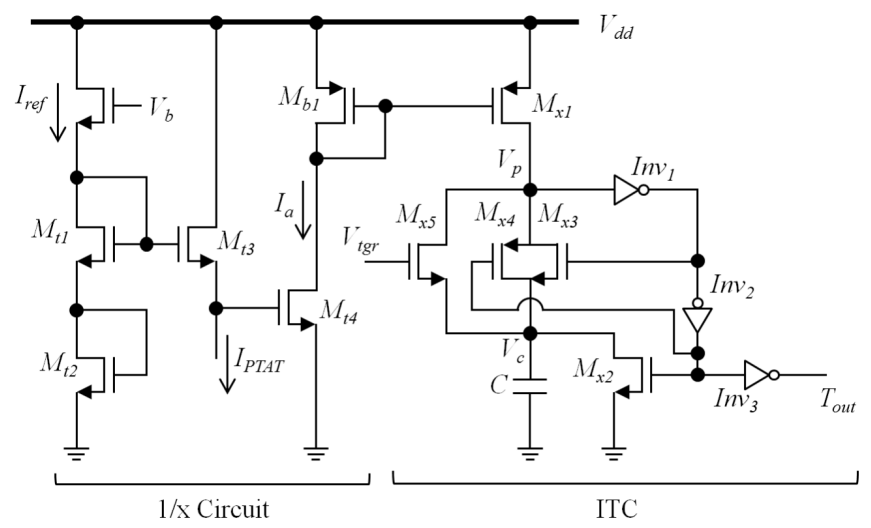

Figure 4. Current to Time Converter (ITC)

The operating principle of ITC could be described as follows, When $V_{t g r}$ shown in ITC circuit is low, $M_{x 3}$, $M_{x 4}$, and $M_{x 5}$ are OFF, and the circuit is in the idle mode. Therefore, $V_{p}$ reaches $V_{d d}$, as the result, $T_{\text {out }}$ becomes Low. When a short single pulse is applied to the gate of $M_{x 5}$, not only $M_{x 5}$ but also $M_{x 3}$ and $M_{x 4}$ become ON, 
because $V_{p}$ becomes Low. As the result, $I_{a}$ flow in $C$, and $C$ store the charges that are proportional $I_{a}$. During this period, $T_{\text {out }}$ is high. The capacitor $C$ is continuously charged, and $V_{p}$ is increasing. When $V_{p}$ reaches the threshold voltage of $I n v_{1}\left(V_{i n v 1}\right), T_{o u t}$ is low, $M_{x 3}$ and $M_{x 4}$ become OFF and $M_{x 2}$ becomes ON, respectively. Therefore, the charge of $C$ is discharged through $M_{x 2}$, and then the circuit returns to the idle mode. Using (16), $T_{\text {out }}$ can be given by

$$
T_{\text {out }}=\frac{C V_{\text {inv1 } 1}}{I_{\text {ref }}^{2}} I_{P T A T}
$$

From (17), it can be inferred that $T_{\text {out }}$ is proportional to $I_{P T A T} . T_{o u t}$ can be converted to the digital value by counting up the period of high level in $T_{\text {out }}$ by a counter.

\subsection{Programmable Temperature Range Digitalization}

In the sub section 2.3 it has been explained that digital value of the measured temperature can be obtained by counting up the high level period of $T_{\text {out }}$ by a counter. However, if $T_{\text {out }}$ is directly connected to counter, it will be ineffective. Because it is known from equation (17) that $T_{\text {out }}$ is proportional to absolute temperature. It means that the high level of $T_{\text {out }}$ will appear as far as the temperature is larger than absolute zero. In the other hand, it is well known that in the biomedical applications, the measured temperature is much higher than absolute zero, for example the critical human body temperature is $35^{\circ} \mathrm{C}$ (hypothermia) to $41.5{ }^{\circ} \mathrm{C}$ (hyperpyrexia). Thus, if $T_{\text {out }}$ is directly connected to counter, the temperature range of digital conversion result will be difficult to be adjusted. Moreover, the digital conversion will not reach high resolution.

In $T_{\text {out }}$-counter direct connection, the temperature range can be adjusted using clock frequency of the counter. The higher clock frequency of counter, the more narrow temperature range that can be convert to digital data. Oppositely to the temperature range, the resolution of digital data is higher. However, for narrow temperature range biomedical applications, $T_{\text {out }}$-counter direct connection is still not effective way to be used. Because $T_{\text {out }}$ pulse width is start to appear right after absolute zero temperature, and increases proportionally as temperature increases. Therefore, if the minimum temperature of the biomedical applications is much larger than absolute zero, the unnecessary conversion will exist. This problem will also make the adjustment difficult, because the counter will be overflow many times before start to converts desired $T_{\text {out }}$ pulse width.

Figure 5 shows the desired conversion and unnecessary conversion in $T_{\text {out }}$ pulse width. To make easier temperature range adjustment and more effective conversion, unnecessary conversion must be eliminated. In other word, the counter counting up only in desired conversion region in $T_{\text {out }}$ pulse width. To perform this function, the connection between $T_{\text {out }}$ and counter is modified. This architecture is shown in Fig. 6.

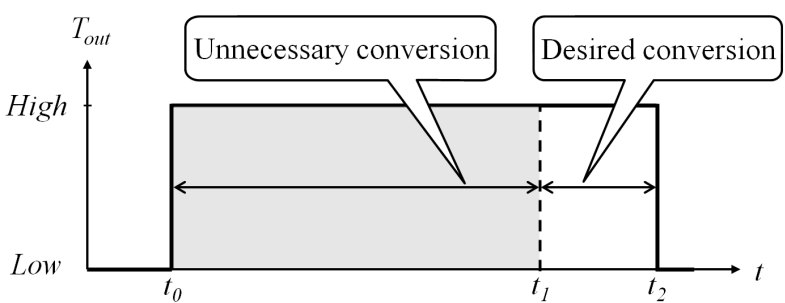

Figure 5. Desired conversion in $T_{\text {out }}$ pulse width

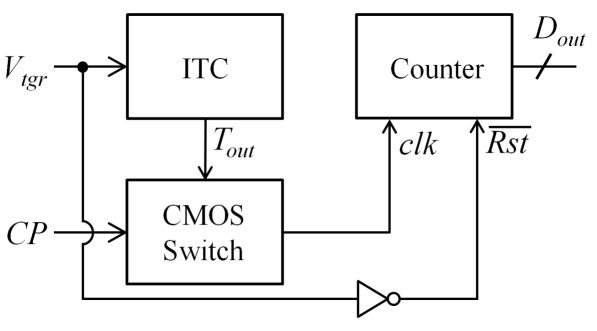

Figure 6. ITC-Counter connection architecture

Using architecture in Fig. 6, the temperature range can be programmed or adjusted not only by clock frequency of the counter but also by pulse width of the trigger $\left(V_{t g r}\right)$. Since $V_{t g r}$ is connected to $\overline{R s t}$ using an inverter, the counter will be in reset condition and it does not count up as far as the $V_{t g r}$ is high. Thus, the conversion only in desired temperature range can be performed by keeping high $V_{t g r}$ from $t_{0}$ to $t_{1}$ shown in Fig. 5. The pulse width of $V_{t g r}$ is written in equation (18). The clock frequency of the counter then can be calculated using equation (19).

$$
\begin{gathered}
V_{t g r_{-} P W}=t_{1}-t_{0} \\
f_{c l k}=\frac{2^{N}-1}{t_{2}-t_{1}}
\end{gathered}
$$

Where $t_{1}$ is pulse width of minimum temperature in temperature range, $t_{2}$ is pulse width of maximum temperature in temperature range, and $N$ is resolution of the counter (bit). Generating pulse and clock with various 
width and frequency is an easy thing that can be performed in programmable devices, like microcontroller and microprocessor. In other word, the temperature range of the proposed temperature sensor is easily programmable, so that it can be used in various biomedical applications.

\section{SIMULATION RESULTS AND MEASUREMENT OF FABRICATED TEMPERATURE SENSOR CORE}

The performance of the proposed circuit was evaluated using HSPICE with 1P, 5M, 3-well, 0.18-m CMOS process (BSIM3v3.2 LEVEL53). All simulations use $1.0 \mathrm{~V}$ supply voltage. Figure 7 shows the simulation result of sensor core circuit in the temperature range of $-40{ }^{\circ} \mathrm{C}$ to $160{ }^{\circ} \mathrm{C}$. $V_{P T A T 1}$ is output of single sensor core, and $V_{P T A T 2}$ is the output of cascade connection of two sensor cores. From this simulation, it was obtained that sensitivity of single sensor core is $0.392 \mathrm{mV} / \mathrm{C}$ with $0.78 \%$ nonlinearity. This sensitivity could be increased using cascade connection like shown by $V_{P T A T 2}$, its sensitivity is $0.783 \mathrm{mV} /{ }^{\circ} \mathrm{C}$, with $0.89 \%$ nonlinearity.

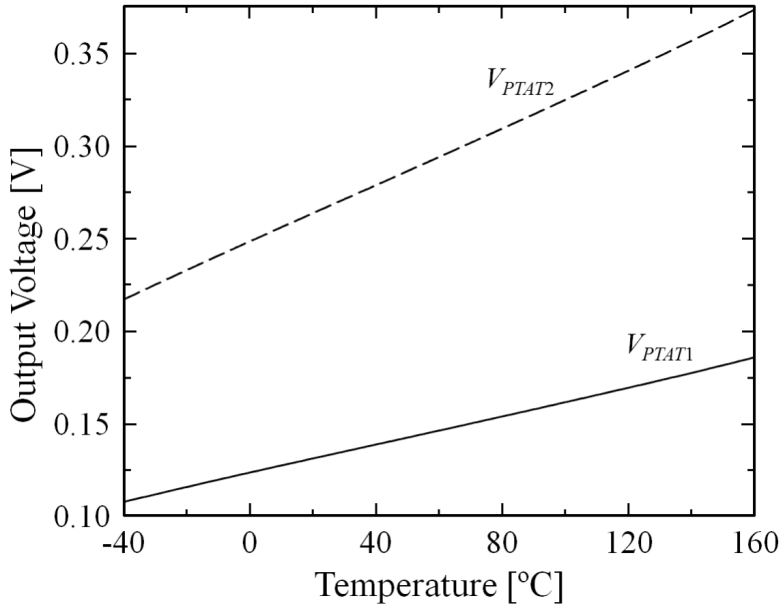

Figure 7. $V_{P T A T}$-Temperature characteristic

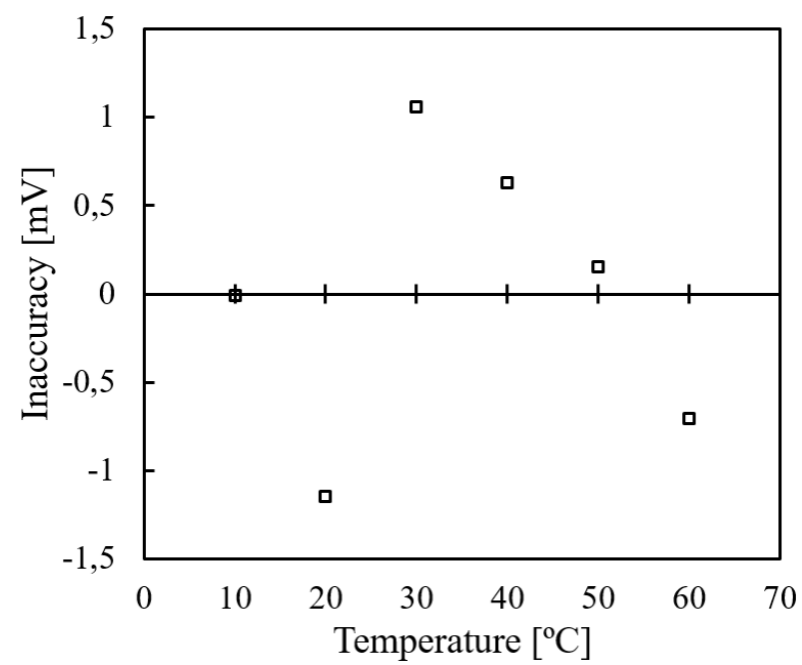

Figure 9. Inaccuracy of fabricated sensor core

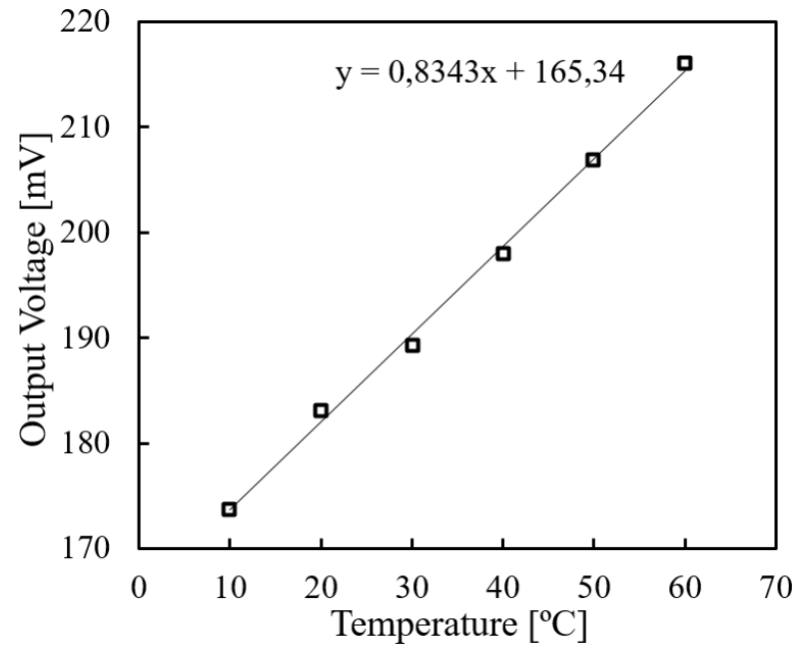

Figure 8. Measurement result of fabricated sensor core

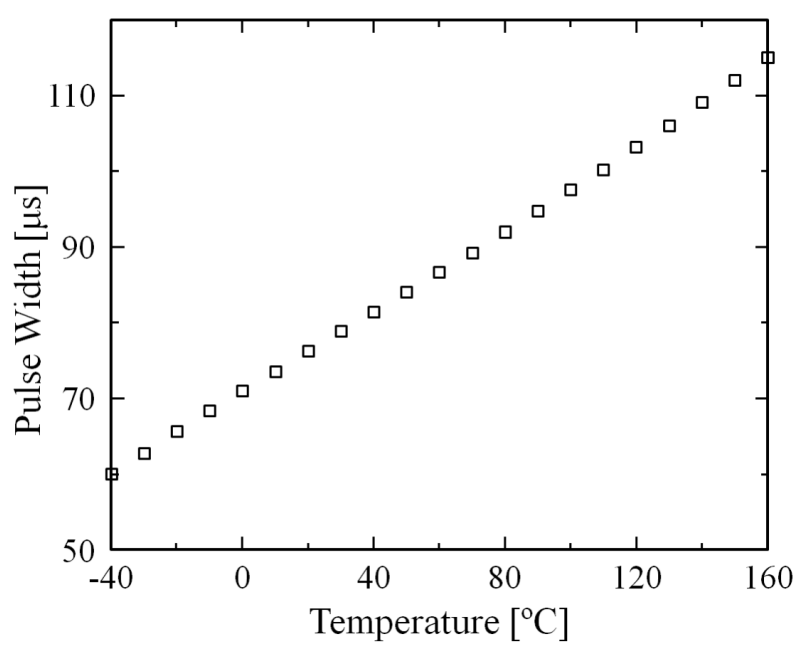

Figure 10. Pulse width-temperature characteristic

In order to verify the performance of temperature sensor core, this temperature sensor core is fabricated using $0.6 \mu \mathrm{m}$ CMOS process. Figure 8 shows the average measurement results of 10 different chips. From this figure it can be known that the output voltage of the temperature sensor core is proportional to temperature. The average sensitivity of 10 measured chips is $0.8343 \mathrm{mV} /{ }^{\circ} \mathrm{C}$. Figure 9 shows the accuracy of fabricated temperature sensor core. From this measurement it was obtained that its inaccuracy is $-1.144 /+1.059 \mathrm{mV}$ or $2.70 \%$ nonlinearity.

Figure 10 shows the relationship between temperature and pulse width. In these simulations, temperature 
Table 1. A comparison of the main performance parameters of temperature sensor circuit

\begin{tabular}{c|c|c|c|c|c|c}
\hline Parameter & This work & {$[4]$} & {$[5]$} & {$[16]$} & {$[17]$} & {$[18]$} \\
\hline Power Supply $(\mathrm{V})$ & 1.0 & 3.3 & $0.5,1.0$ & $2.2-3$ & 3.3 & 1.0 \\
Power consumption $(\mu \mathrm{W})$ & 22.3 & 429 & 0.119 & $10-27$ & 10 & 25 \\
Range $\left({ }^{\circ} \mathrm{C}\right)$ & programmable & -50 to 125 & -10 to 30 & 10 to 80 & 0 to 100 & 50 to 120 \\
Inaccuracy $\left({ }^{\circ} \mathrm{C}\right)$ & -0.98 to +0.29 & -0.5 to +0.5 & -0.8 to +1.0 & -1.8 to +1.0 & -0.7 to +0.9 & -1.0 to +0.8 \\
Process $(\mu \mathrm{m})$ & 0.18 & 0.5 & 0.18 & 0.35 & 0.35 & 0.09 \\
\hline
\end{tabular}

was changed from $-40{ }^{\circ} \mathrm{C}$ to $160^{\circ} \mathrm{C}$ in step of $10^{\circ} \mathrm{C}$. As a result, it was obtained that the pulse width was proportional to temperature with $0.276 \mu \mathrm{s} /{ }^{\circ} \mathrm{C}$ sensitivity and $2.14 \%$ nonlinearity. Based on the data from these simulations, temperature range programmability of this proposed circuit is verified by programming its temperature range to be $0{ }^{\circ} \mathrm{C}$ to $100{ }^{\circ} \mathrm{C}$. Using equations (18) and (19) the pulse width of $V_{t g r}$ and clock frequency of the counter $(C P)$ are $70.9 \mu$ s and $9.59 \mathrm{MHz}$, respectively.

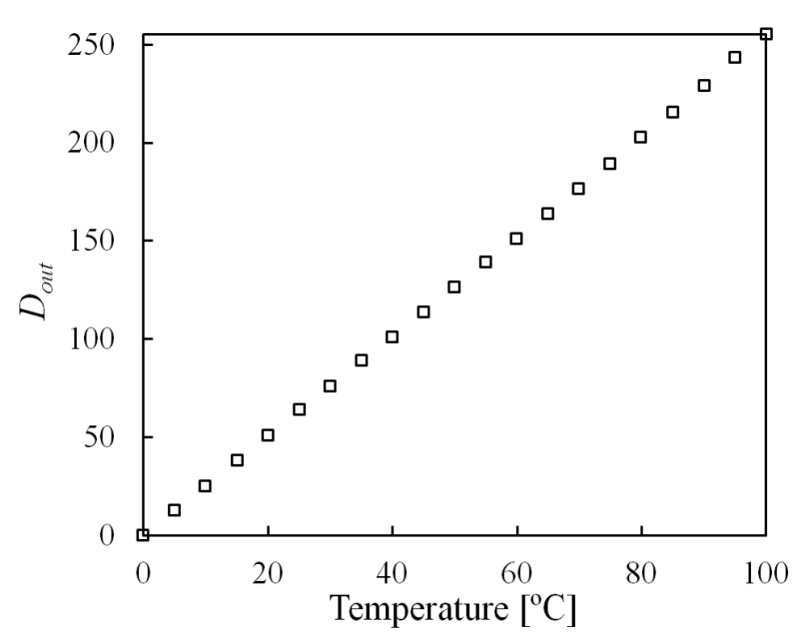

Figure 11. $\mathrm{D}_{\text {out }}$-temperature characteristic

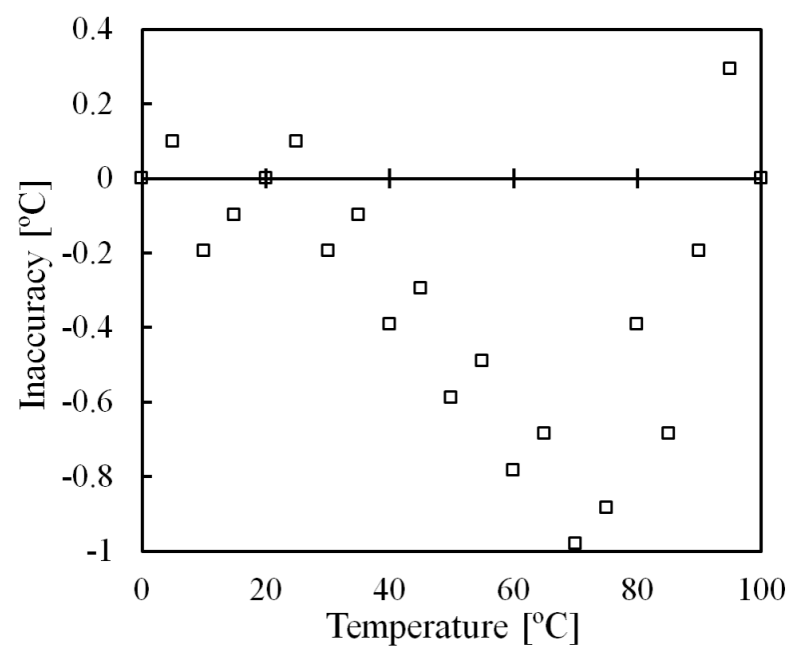

Figure 12. Inaccuracy of the proposed temperature sensor

Figure 11 shows digital output of the simulation result after the temperature range of the sensor is programmed to be $0{ }^{\circ} \mathrm{C}$ to $100{ }^{\circ} \mathrm{C}$. From the simulation it was obtained that digital code $D_{\text {out }}$ is proportional to temperature. The digital code of this simulation was 0 to 255 . This means that 1 LSB is equal to $0.392{ }^{\circ} \mathrm{C}$. Figure 12 shows the accuracy of the proposed integrated temperature sensor circuit. The accuracy measurement was done using two calibration points $\left(0^{\circ} \mathrm{C}\right.$ and $\left.100{ }^{\circ} \mathrm{C}\right)$. From this measurement it was obtained that inaccuracy of the proposed circuit is $-0.98 /+0.29^{\circ} \mathrm{C}$. From these simulation results it can be verified that the temperature range of the sensor can be programmed well.

The power consumption of the proposed circuit in $25{ }^{\circ} \mathrm{C}$ is $22.3 \mu \mathrm{W}$. This value is sum of all circuits, sensor core $(1.72 \mu \mathrm{W})$, VIC $(1.21 \mu \mathrm{W}), 1 / \mathrm{x}$ circuit $(0.331 \mu \mathrm{W})$, ITC $(1.16 \mu \mathrm{W})$, and counter $(17.8 \mu \mathrm{W})$. Lastly, a comparison of main performance parameters of temperature sensor is summarized in Table 1.

\section{CONCLUSION}

In this paper, the integrated temperature sensor circuit is constructed using sensor core, voltage-to-current converter (VIC), 1/x circuit, current-to-time converter (ITC) and counter. Sensor core is formed using CMOS circuit operated in weak inversion region and it is insensitive to device parameter of fabrication process. The output of the sensor is then digitized using proposed programmable temperature range digital interface.

The performance of the proposed circuit was evaluated using HSPICE with 1P, 5M, 3-wells, 0.18-m CMOS process (BSIM3v3.2 LEVEL53). As a result, sensitivity of temperature sensor core is $0.783 \mathrm{mV} /{ }^{\circ} \mathrm{C}$, with $0.89 \%$ nonlinearity in $-40{ }^{\circ} \mathrm{C}$ to $160{ }^{\circ} \mathrm{C}$. This temperature sensor core has been fabricated using $0.6 \mu \mathrm{m}$ CMOS process. As a result of 10 different chips measurement is the average sensitivity of fabricated chip is $0.8343 \mathrm{mV} /{ }^{\circ} \mathrm{C}$ in $10{ }^{\circ} \mathrm{C}$ to $60{ }^{\circ} \mathrm{C}$, with $2.70 \%$ nonlinearity.

Temperature range of the sensor is then programmed to be $0{ }^{\circ} \mathrm{C}$ to $100{ }^{\circ} \mathrm{C}$ using pulse width of $V_{t g r}$ and 
clock frequency of counter $(C P)$. As the results of simulation, resolution of the sensor that its temperature range has been programmed is $0.392{ }^{\circ} \mathrm{C}$ with $-0.98 /+0.29{ }^{\circ} \mathrm{C}$ inaccuracy and total power consumption is $22.3 \mu \mathrm{W}$ in $25^{\circ} \mathrm{C}$.

The future work of this research is designing mask layout of the proposed digital interface, fabrication of whole circuit in one prototype chip and evaluation of the characteristic.

\section{ACKNOWLEDGEMENT}

This work is supported by VLSI Design and Education Center (VDEC), the University of Tokyo in collaboration with Synopsys, Inc. and Cadence Design Systems, Inc.

\section{References}

[1] A. Goel, and G. Singh, "A Novel Low Noise High Gain CMOS Instrumentation Amplifier for Biomedical Applications," International Journal of Electrical and Computer Engineering (IJECE), Vol. 3, No. 4, pp. 516523, Aug. 2013.

[2] S. Meti, and V.G. Sangam, "A Thorough Insight to Techniques for Performance Evaluation in Biological Sensors," International Journal of Electrical and Computer Engineering (IJECE), Vol. 6, No. 3, pp. 986-994, Jun. 2016.

[3] X. Zhang, H. Zhang, G. Kang, P. Zhang, and H. Li, "External Biomedical Device Relaying Body Sensor Network scheme," TELKOMNIKA, Vol. 11, No. 12, pp. 71027109, Dec. 2013.

[4] M. A. P. Pertijs, A. Niederkon, X. Ma, B. McKillop, A. Bakker, and J. H. Huijsing, "A CMOS Temperature Sensor With a 3 Inaccuracy of 0.5 C From 50 C to 120 C," IEEE JOUNAL OF SOLID-STATE CIRCUITS, Vol. 40, No. 2, pp. 454460, Feb. 2005.

[5] Z. K. Law, A. Bermak and H. C. Luong: "A Sub-W Embedded CMOS Temperature Sensor for RFID Food Monitoring Application", IEEE Journal of Solid-State Circuits, VOL. 45, NO. 6, pp. 12461255, Jun. 2010.

[6] R. Sakamoto, K. Tanno, H. Tamura and Zainul Abidin, ”A Sub-W, 1.0 V CMOS Temperature Sensor Circuit Insensitive to Device Parameters," IEEE region 10 conference TENCON 2011, pp.626-629, Nov. 2011.

[7] P. C. Crepaldi, T. C. Pimenta, and R. L. Moreno, "A CMOS low-voltage low-power temperature sensor," Microelectronics Journal, Vol. 41, No. 9, pp. 594600, June 2010.

[8] J. Fujitsuka and K. Takakubo, "A Consideration of Temperature Coeffi-cient on Gate-Voltage-Controlled PTAT Voltage Generator under Ultra Low Power Supply," Proc. of Electronics, Information and Systems Conference, Electronics, Information and Systems, I.E.E of Japan, GS11-2, pp. 14521457, Sep. 2010.

[9] K. Tanno, T. Makoto, H. Tamura, and O. Ishizuka, "High-Performance CMOS Temperature Sensor Circuit," Note on Multiple-Valued logic in Japan, Vol. 32, No. 9, pp. 16, Sep. 2009.

[10] T. Ohzone, T. Sadamoto, T. Morishita, K Komoku, T. Matsuda, and H. Iwata, "A CMOS Temperature Circuit," IEICE TRANS. ELECTRON, Vol. E90-C, No. 4, pp. 895902, Apr. 2007.

[11] M. Tuthill, "A Switched-Current, Switched-Capacitor Temperature Sensor in 0.6-m CMOS," IEEE JOUNAL OF SOLID-STATE CIRCUITS, Vol. 33, No. 7, pp. 11171122, Jul. 1998.

[12] K. S. Szajda, C. G. Sodini, and H. F. Bowman, "A Low Noise, High Resolution Silicon Temperature Sensor," IEEE JOUNAL OF SOLID-STATE CIRCUITS, Vol. 31, No. 9, pp. 13081313, Sep. 1996.

[13] H. Ikeda, K. Takakubo, and H. Takakubo, "Drain Current Zero-Temperature-Coefficient Point for CMOS Temperature-Voltage Converter Operating in Strong Inversion,' IEICE TRANS. FUNDAMENTALS, Vol. E87A, No. 2, pp. 370375, Feb. 2004.

[14] A. Setiabudi, R. Sakamoto, H. Tamura and K. Tanno, "A Low-Voltage and Low-Power CMOS Temperature Sensor Circuit with Digital Output for Wireless Healthcare Monitoring System," 2016 IEEE 46th International Symposium on Multiple-Valued Logic (ISMVL), Sapporo, pp. 183-188, 2016.

[15] R. Sakamoto, K. Tanno, H. Tamura, ”A Low-Power and High-Linear Current to Time Converter for Wireless Sensor Networks," IEICE TRANSACTIONS on Fundamentals of Electronics, Communications and Computer Sciences, Vol.E95-A No.6 pp.1088-1090, 2012.

[16] K. Ueno, T. Asai and Y. Amemiya, "Low-power temperature-to-frequency converter consisting of subthreshold CMOS circuits for integrated smart temperature sensors," Elsevier Sensor and Actuators A: Physical, Vol. 165, Issue. 1, pp. 132-137, Jan 2011.

[17] P. Chen, C. C. Chen, C. C. Tsai and W. F. Lu, "A Time-to-Digital-Converter-Based CMOS Smart Temperature Sensor," IEEE J. Solid-State Circuits, Vol. 40, No. 8, pp. 1642-1648, Aug. 2005.

[18] M. Sasaki, M. Ikeda and K. Asada, "A Temperature Sensor with an Inaccuracy of -1/+0.8 C using 90-nm 1-V CMOS for Online Thermal Monitoring of VLSI Circuits,' IEEE Trans. Semiconductor Manufacturing, Vol. 21, No. 2, pp. 201-208, May 2008. 